\title{
Catheter ablation of atrial fibrillation after pericardiectomy: multi- center experience in China
}

\author{
Ronghui Yu ${ }^{1,2 \#}$, Hui $\mathrm{Xi}^{3 \#}$, Peize Wang ${ }^{1,2}$, Dongling $\mathrm{Xu}^{4}$, Jun $\mathrm{Lu}^{5}$, Fengqiang $\mathrm{Xu}^{5}$, Lei $\mathrm{An}^{6}$, Xin $\mathrm{Zhao}^{1,2}$, \\ Rong Bai ${ }^{1,2}$ \\ ${ }^{1}$ Department of Cardiology, Beijing Anzhen Hospital, Capital Medical University, Beijing 100029, China; ${ }^{2}$ National Clinical Research Center for \\ Cardiovascular Diseases, Beijing 100029, China; ${ }^{3}$ Department of Cardiology, Peking University International Hospital, Beijing 102206, China; \\ ${ }^{4}$ Department of Cardiology, The Second Hospital of Shandong University, Jinan 250033, China; ${ }^{5}$ Department of Cardiology, The Affiliated Hospital \\ of Qingdao University, Qingdao 266000, China; ${ }^{6}$ Department of Cardiology, Langfang People Hospital, Langfang 250033, China \\ Contributions: (I) Conception and design: R Yu; (II) Administrative support: P Wang, Hui Xi; (III) Provision of study materials or patients: P Wang, \\ H Xi, D Xu, J Lu, F Xu, L An; (IV) Collection and assembly of data: X Zhao, R Bai; (V) Data analysis and interpretation: R Yu, X Zhao, R Bai; (VI) \\ Manuscript writing: All authors; (VII) Final approval of manuscript: All authors. \\ \#These authors contributed equally to this work. \\ Correspondence to: Ronghui Yu. Department of Cardiology, Beijing Anzhen Hospital, Capital Medical University, Beijing 100029, China; National \\ Clinical Research Center for Cardiovascular Diseases, Beijing 100029, China. Email: ronghuiyu20@163.com.
}

Background: To study the effectiveness and safety of atrial fibrillation (AF) catheter ablation after pericardiectomy.

Methods: Data of 24 consecutive AF patients after pericardiectomy underwent catheter ablation from five centers were collected and analyzed retrospectively. All patients were followed up at 1,3, and 12 months after catheter ablation. Results of a repeated blood test, electrocardiogram, and echocardiography during followup were also collected. Adverse events such as recurrence of AF, heart failure, stroke/transient ischemic attack (TIA) and minor, and major bleeding were recorded. All patients underwent brain magnetic resonance imaging (MRI) at the end of 12 months follow-up.

Results: Patients were young (20-73 years old, 48.1 \pm 11.0$)$. Fifteen (62.5\%) patients were male. CHA2DS2VASc score $(0-3,0.21 \pm 0.41)$ was low in these 24 patients. Among these patients, $11(45.8 \%)$ were paroxysmal $\mathrm{AF}, 8(33.3 \%)$ were persistent $\mathrm{AF}$, and 5 (20.8\%) were long-lasting persistent AF. Left atrium diameter over $45 \mathrm{~mm}$ was detected in 17 (70.8\%) patients. All patients underwent catheter ablation successfully. No periablation procedure-related complication happened. Oral anticoagulant therapy was stopped 3 months after the final ablation. Anti-arrhythmia drugs were continued for all patients after ablation. For 12 months follow-up, $\mathrm{AF}$ recurred in $10(41.7 \%)$ patients $3-7$ months after the first ablation. MRI detected silent cerebral infarction (SCI) in 2 (8.3\%) patients. No other adverse events occurred during follow-up.

Conclusions: It is safe for $\mathrm{AF}$ patients to undergo catheter ablation after pericardiectomy, but the rate of recurrence of $\mathrm{AF}$ is high.

Keywords: Atrial fibrillation (AF); catheter ablation; pericardiectomy

Submitted Feb 11, 2020. Accepted for publication Mar 07, 2020.

doi: $10.21037 /$ atm.2020.04.49

View this article at: http://dx.doi.org/10.21037/atm.2020.04.49

\section{Introduction}

Atrial fibrillation (AF) is often seen in clinical practice in China (1-3). It has an adverse influence on patients' heart function and quality of life and increases the risk of stroke, transient ischemic attack (TIA), and even death (4). In the past, heart rate control and conversion to sinus rhythm were both first line choice for patients with AF (5). With the rapid progress of catheter ablation for AF, researchers 
gradually realized that conversion to sinus rhythm was before heart control for long-term prognosis (6). In recent years, increased patients with AF underwent catheter ablation, including radiofrequency ablation, cryoballoon ablation, and hot balloon ablation. Post-ablation anticoagulation, $\mathrm{AF}$ recurrence, and dementia are focuses of study nowadays (7-9). Patients with constrictive pericarditis often suffer AF because of the limitation of left ventricular movement (10). Though pericardiectomy can release the over-pressure of heart, it cannot convert AF to normal sinus rhythm (10). It is also necessary for patients who received pericardiectomy before to undergo catheter ablation to reduce adverse events and improve heart function and quality of life. But few studies had focused on this patient, and clinical experience was insufficient. In this study, we summarize and analyzed clinical data of patients from five centers in China to provide preliminary experience, including the safety and effectiveness of ablation and midto long-term prognosis.

\section{Methods}

\section{Study patients and definitions}

We enrolled patients (observation group, OG) from five centers in China between Mar 2015 and Jun 2019 according to inclusion criteria and exclusion criteria. Inclusive criteria: underwent pericardiectomy before catheter ablation; underwent catheter ablation only for AF; age over 18 years old. Exclusive criteria: connected tissue disease; malignant tumor; pregnancy. Paroxysmal AF refers to AF that terminates spontaneously; persistent $\mathrm{AF}$ refers to $\mathrm{AF}$ that sustained beyond 7 days; long-standing persistent AF refers to $\mathrm{AF}$ that continued beyond 1 year $(11,12)$. Silent cerebral infarction (SCI) refers to the presence of focal cerebral ischemia in the absence of clinical signs and symptoms (13). After enrollment, we enrolled AF patients who received catheter ablation during the same period as the control group (CG). These patients had no history or evidence of pericarditis or pericardiectomy. This study was approved by the ethics committee of each hospital respectively.

\section{Catheter ablation procedure}

All patients underwent catheter ablation radiofrequency. In these five centers, the process of catheter ablation was similar and conducted according to guidelines $(14,15)$ and all patients signed informed content. In short, amiodarone was stopped for at least 1 month before ablation, and other anti-arrhythmia drugs were suspended for at least five halflife periods. Transesophageal echocardiography was used to detect thrombosis in the left atrium before ablation. After local anesthesia with $1 \%$ lidocaine, an $8.5-\mathrm{F}$ - and a 6-F-sheath sheath was implanted into the right femoral vein. The mapping electrode was inserted into the coronary sinus through 6-F-sheath. After atrial septum was punctured, an ablation catheter (ThermoCool SmartTouch, Biosense Webster, Diamond Bar, CA, USA) was advanced to the left atrium. The radiofrequency ablation and $3 \mathrm{D}$ model were conducted under the EAM system (CARTO3, Biosense Webster, Diamond Bar, CA, USA). For paroxysmal AF, after high-density mapping of the left atrium, continuous circumferential ablation around the ipsilateral pulmonary vein cavity was performed to isolate all pulmonary veins (Figure 1) completely. For persistent AF, in addition to pulmonary vein isolation, linear ablation at the left atrium roof (LAF), mitral isthmus (MI), and cavotricuspid isthmus (CTI) where required. If the patient did not convert to sinus rhythm after ablation, cardioversion was conducted to convert AF to sinus rhythm (16).

\section{Data collection}

Patients' information including sex, age, personal history, physical examination, blood test, electrocardiogram (ECG) and echocardiography results on the admission of hospitalization for catheter ablation, type of AF, catheter ablation procedural parameters were all collected from in-hospital records.

\section{Post-ablation follow-up}

After successful catheter ablation, all patients were followed up for at least 12 months. Clinic visit was needed at 1-, 3 -, and 12-month follow-up. Oral anticoagulation (OAC) therapy was stopped 3 months after ablation if the patient's $\mathrm{CHA}_{2} \mathrm{DS}_{2}$-VASc score was not over 2. If AF recurred, OAC therapy and antiarrhythmic drug (AAD) has continued again. Blood tests, ECGs, and echocardiography were all repeated for each patient at follow-up. At the end of the 12-month follow-up, all patients received a brain magnetic resonance imaging (MRI) examination.

\section{Statistical analysis}

Continuous variables were expressed as the mean \pm standard deviation (SD) and compared using an unpaired two- 

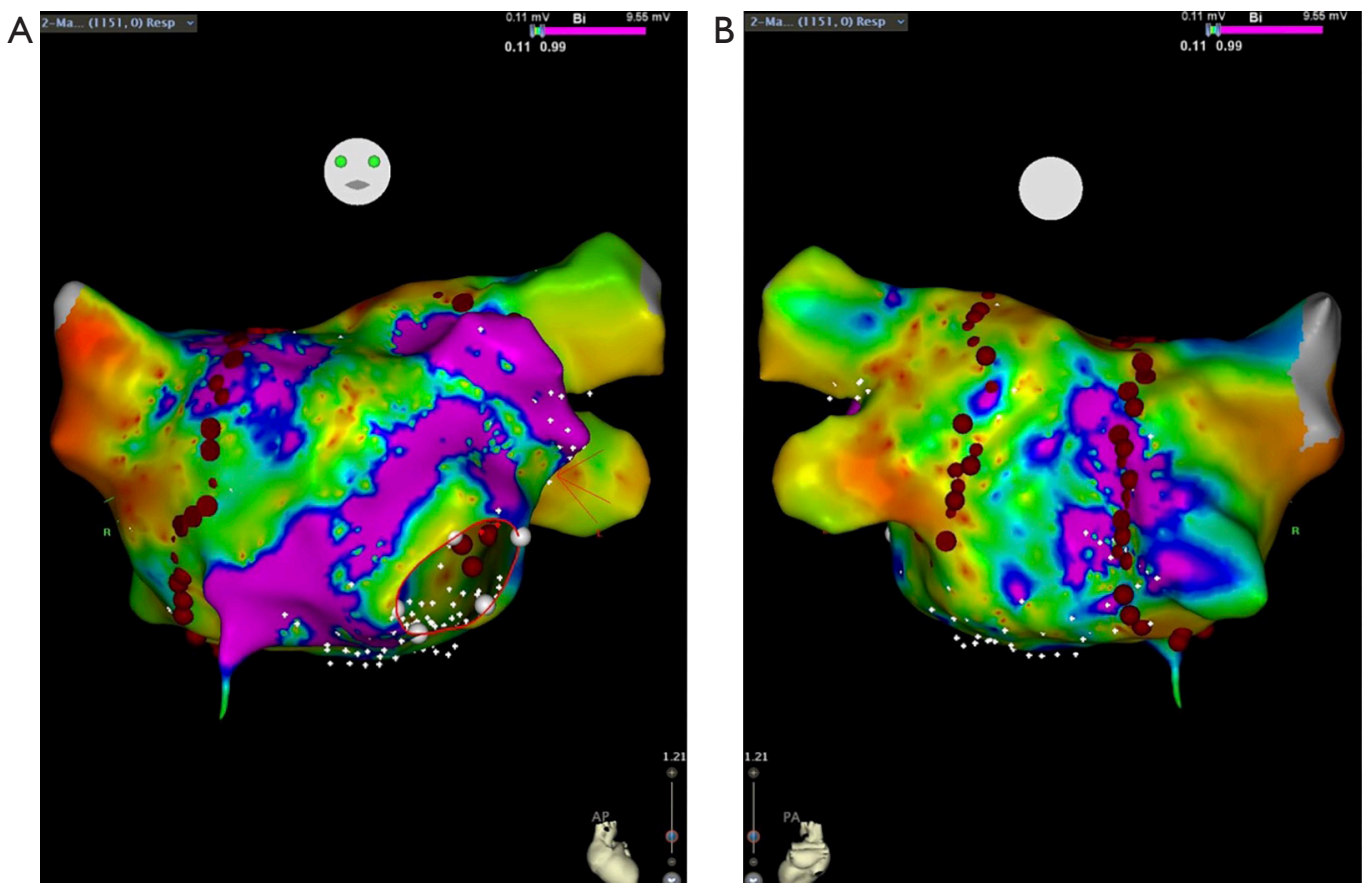

Figure 1 High-density mapping and quantitative ablation of atrial fibrillation after pericardiectomy. Under voltage mapping, the voltage value of the pulmonary vein antrum was low. (A) Anterior-posterior projection; (B) posterior-anterior projection.

sided Student's $t$-test when normal distribution and equal dispersion were confirmed. The Mann-Whitney $U$ test and the Wilcoxon's signed-rank test were used when the variance was unequal. Categorical variables were expressed as percentages (\%) and compared using $\chi^{2}$ analysis or Fisher's exact test if necessary. A P value of less than 0.05 was considered statistically significant. All statistical analysis was performed with SPSS (version 17.0 for Windows, SPSS, Inc., Chicago, IL, USA).

\section{Results}

\section{Baseline characteristics}

According to inclusive and exclusive criteria, 24 consecutive patients were enrolled in the final analysis. Fifteen $(62.5 \%)$ patients were male. Among these patients, 11 (45.8\%) were paroxysmal AF, 8 (33.3\%) were persistent $\mathrm{AF}$, and $5(20.8 \%)$ were long-standing persistent AF. Left atrium diameter (LAd) over $45 \mathrm{~mm}$ was detected in 17 (70.8\%) patients. The CG enrolled 1,504 patients. When compared with patients in the CG, patients in OG were relatively young (2073 years old, $48.1 \pm 11.0$ ), and their SBP and DBP were all lower than those in CG. The morbidity rates of hypertension, hyperlipidemia was lower in OG. Three patients were over 60 years old. CHA2DS2-VASc score $(0-2,0.21 \pm 0.41)$ was relatively small in these 24 patients. The time between pericardiectomy and first catheter ablation was 4 to 21 months $(10.2 \pm 4.7)$. Before catheter ablation, only three patients were administrated with new OAC (NOAC), and 11 patients were administrated with aspirin, and the remain 10 patients had not received any anti-thrombosis medication, details were listed in Table 1 .

\section{Results of catheter ablation}

All patients underwent radiofrequency catheter ablation successfully. Twenty patients received successful catheter ablation at the first procedure, and the other four patients were treated successfully at the second procedure. Eleven patients with paroxysmal AF received circumferential pulmonary vein isolation (CPVI). Thirteen persistent AF patients (including long-standing) received CPVI + $\mathrm{LAF}+\mathrm{MI}+\mathrm{CTI}$. No peri-ablation procedure-related complication happened. As we can see from Table 2, compared with CG, OG had more ablation points and longer procedural time. 
Table 1 Baseline characteristics in OG and comparison with CG

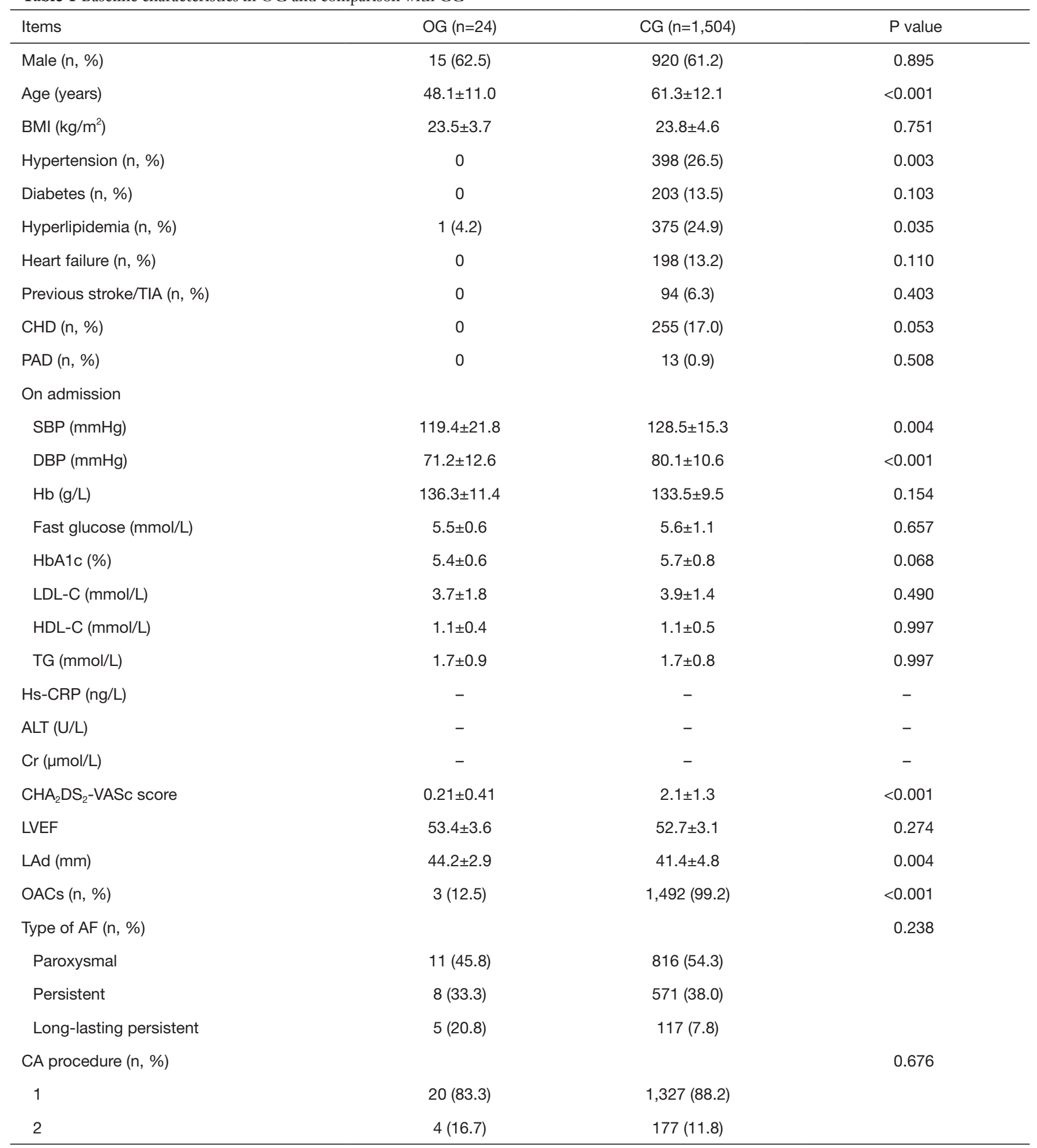

OG, observation group; CG, control group; BMI, body mass index; TIA, transient ischemic attack; CHD, coronary heart disease; PAD, peripheral artery disease; SBP, systolic blood pressure; DBP, diastolic blood pressure; Hb, hemoglobin; LDL-C, low density lipoprotein cholesterol; HDL-C, high density lipoprotein cholesterol; TG, triglyceride; hs-CRP, high sensitivity C-reactive protein; ALT, alanine aminotransferase; Cr, creatine; LVEF, left ventricular ejection fraction; LAd, left atrium diameter; AF, atrial fibrillation; OAC, oral anticoagulant; $\mathrm{CA}$, catheter ablation. 
Table 2 Parameters of electrophysiological examination and catheter ablation

\begin{tabular}{lccc}
\hline Items & OG $(\mathrm{n}=24)$ & $\mathrm{CG}(\mathrm{n}=504)$ & $\mathrm{P}$ value \\
\hline Volume of left atrium reconstruction $(\mathrm{mL})$ & $148.23 \pm 59.07$ & $132.23 \pm 40.23$ & 0.064 \\
Voltage of pulmonary vein antrum $(\mathrm{mV})$ & $0.81 \pm 0.72$ & $1.14 \pm 0.80$ & 0.048 \\
Ablation points $(\mathrm{n})$ & $98 \pm 43$ & $79 \pm 39$ & 0.026 \\
Procedural time $(\mathrm{min})$ & $146 \pm 24$ & $118 \pm 37$ & 0.0001 \\
Fluoroscopy time $(\mathrm{min})$ & $15 \pm 6$ & $14 \pm 7$ & 0.492 \\
\hline
\end{tabular}

OG, observation group; CG, control group.

Table 3 Outcome at follow-up

\begin{tabular}{lccc}
\hline Items & $\mathrm{OG}(\mathrm{n}=24)$ & $\mathrm{CG}(\mathrm{n}=1,504)$ & $\mathrm{P}$ value \\
\hline AF recurrence $(\mathrm{n}, \%)$ & $10(41.7)$ & $203(13.5)$ & 0.0001 \\
$\begin{array}{l}\text { Pericardial effusion } \\
(\mathrm{n}, \%)\end{array}$ & 0 & $2(0.1)$ & $>0.05$ \\
$\begin{array}{l}\text { Stroke/TIA (n, \%) } \\
\text { Heart failure (n, \%) }\end{array}$ & 0 & $35(2.3)$ & $>0.05$ \\
$\begin{array}{l}\text { Cerebral hemorrhage } \\
(\mathrm{n}, \%)\end{array}$ & 0 & $17(1.1)$ & $>0.05$ \\
Death $(\mathrm{n}, \%)$ & $23(1.5)$ & $>0.05$ \\
\end{tabular}

OG, observation group; CG, control group; TIA, transient ischemic attack.

\section{Follow-up}

For 12 months follow-up, AF recurred in 10 (41.7\%) patients 3-7 months after successful ablation. All patients discontinued OAC therapy on doctors' advice 3 months after successful ablation. SCI was detected by MRI in 2 (8.3\%) patients. Repeated echocardiography at a 3-month follow-up and 12-month follow-up did not find the sign of pericardial effusion, which suggested pericardial hemorrhage. No other adverse events occurred during follow-up. Results of follow-up blood tests, ECG, and echocardiography were shown in Table 3. Incidence of SCI was not compared between OG and CG because not all patients in CG underwent a brain MRI scan.

\section{Discussion}

In this study, we supplied some experience in catheter ablation for AF patients after pericardiectomy. The success rate of the first ablation procedure was $58.3 \%$ though LAd in most patients $(70.8 \%)$ was over $45 \mathrm{~mm}$. The process was safe in our catheter lab except that it needed more ablation points and longer procedural time, which were acceptable, because this kind of patient was younger and had fewer comorbidities, the CHA2DS2-VASc score was also relatively lower.

The primary pathophysiology of constrictive pericarditis is the limitation of left ventricle diastole (17). This leads to the increase of pressure in the left atrium and the expansion of LAd, which is believed to be a strong risk factor of atrial arrhythmia, especially AF (18). Previous studies demonstrated that left atrium developed fibrosis with the expansion of its diameter $(19,20)$, the larger volume of left atrium reconstruction, and the lower voltage value of pulmonary vein antrum in our study confirm it (Figure 1). This histological and cellular remodeling resulted in electrical remodeling, which is the pathophysiological basis of AF (21). Hence, the expansion of the left atrium also increases the difficulty of catheter ablation and is a predictor of procedure failure. In our study, $58.3 \%$ of patients succeeded in the first procedure, which was lower than the CG. This result suggested that it was not so challenging to perform catheter ablation for $\mathrm{AF}$ patients after pericardiectomy. During 12 months of follow-up, 10 (41.7\%) patients suffered again AF diagnosed by regular ECG or 24 hours Holter monitoring. This recurrence rate was also a little high. The reason should be the remodeling of the left atrium. This result suggested that pericardiectomy could only improve the hemodynamics but also the histological and electrical remodeling. Whether cryoballoon ablation and hot balloon ablation have a lower incidence of recurrence or not is still unclear. Further study should focus on the supporting of sinus rhythm and the effectiveness of other type ablation for this kind of patient.

Because of the risk of pericardial hemorrhage, doctors were reluctant to prescribe $\mathrm{OAC}$ to AF patients with pericarditis. Pericardiectomy also puts patients at elevated 
risk of post-operation hemorrhage. And there was a lack of evidence to support the use of OAC in these patients. Guidelines also did not comment on this subject. So, in clinical practice, few patients received OAC. In our present study, only three patients were administrated with NOAC before ablation. Further analysis revealed that these three patients were all over 60 years old, and their $\mathrm{CHA}_{2} \mathrm{DS}_{2}-$ VASc scores were all 2. And these three patients were all under intensive echocardiography monitoring to detect pericardial effusion. Considering the $\mathrm{CHA}_{2} \mathrm{DS}_{2}$-VASc score of our patients in this study was relatively low and not over 2, to prevent thrombosis or hemorrhage events, we administrated OAC only for 3 months and discontinued OAC therapy at the end of 3 months of follow-up. When $\mathrm{AF}$ recurred, OAC was started again. The outcome turned out to be safe and reasonable. This result was consistent with other studies (22). SCI is believed to be strongly related to future stroke. Previous studies reported that the frequency of SCI in patients with AF detected by MRI was $40 \%$ and by computed tomography was $22 \%$ (23). Patients with both SCI and AF were threefold more likely to develop mild cognitive impairment (MCI) than patients with either SCI or AF only (24). At the end of the 12-month followup, we conducted a brain MRI examination and found only $2(8.3 \%)$ patients had SCI, which also suggested a low risk of stroke in these patients. Repeated echocardiography at a 3-month follow-up and 12-month follow-up did not find a sign of pericardial effusion, which showed that there is no pericardial hemorrhage. In summary, we suggested that for this kind of patients, it is safe and effective to administrate OAC for 3 months. What should be pointed out is that the time between pericardiectomy and the first catheter ablation was four to 21 months. So, we still don't know how long the shortest period between pericardiectomy and OAC initiation is. Taking our result into consideration, we recommend at least 3 months before start OAC therapy after pericardiectomy.

\section{Acknowledgments}

Funding: This work was supported by the Beijing Municipal Science and Technology Commission (RY, Z181100001718174).

\section{Footnote}

Conflicts of Interest: All authors have completed the ICMJE uniform disclosure form (available at http://dx.doi. org/10.21037/atm.2020.04.49). JL serves as an unpaid Section Editor of Annals of Translational Medicine from Oct 2019 to Sep 2020. The other authors have no conflicts of interest to declare.

Ethical Statement: The authors are accountable for all aspects of the work in ensuring that questions related to the accuracy or integrity of any part of the work are appropriately investigated and resolved. This study was approved by the ethics committee of each hospital respectively (No. D11110700300000).

Open Access Statement: This is an Open Access article distributed in accordance with the Creative Commons Attribution-NonCommercial-NoDerivs 4.0 International License (CC BY-NC-ND 4.0), which permits the noncommercial replication and distribution of the article with the strict proviso that no changes or edits are made and the original work is properly cited (including links to both the formal publication through the relevant DOI and the license). See: https://creativecommons.org/licenses/by-nc-nd/4.0/.

\section{References}

1. Wang X, Fu Q, Song F, et al. Prevalence of atrial fibrillation in different socioeconomic regions of China and its association with stroke: Results from a national stroke screening survey. Int J Cardiol 2018;271:92-7.

2. Rahman F, Kwan GF, Benjamin EJ. Global epidemiology of atrial fibrillation. Nat Rev Cardiol 2014;11:639-54.

3. Guo Y, Tian Y, Wang H, et al. Prevalence, incidence, and lifetime risk of atrial fibrillation in China: new insights into the global burden of atrial fibrillation. Chest 2015;147:109-19.

4. Jagadish PS, Kirolos I, Khare S, et al. Post-operative atrial fibrillation: should we anticoagulate? Ann Transl Med 2019;7:407.

5. Hucker WJ, Hanley A, Ellinor PT. Improving Atrial Fibrillation Therapy: Is There a Gene for That? J Am Coll Cardiol 2017;69:2088-95.

6. Ecker V, Knoery C, Rushworth G, et al. A review of factors associated with maintenance of sinus rhythm after elective electrical cardioversion for atrial fibrillation. Clin Cardiol 2018;41:862-70.

7. Doherty JU, Gluckman TJ, Hucker WJ, et al. 2017 ACC Expert Consensus Decision Pathway for Periprocedural Management of Anticoagulation in Patients With Nonvalvular Atrial Fibrillation: A Report of the American 
College of Cardiology Clinical Expert Consensus

Document Task Force. J Am Coll Cardiol 2017;69:871-98.

8. Mujović N, Marinković M, Lenarczyk R, et al. Catheter Ablation of Atrial Fibrillation: An Overview for Clinicians. Adv Ther 2017;34:1897-917.

9. Chopard R, Piazza G, Gale SA, et al. Dementia and Atrial Fibrillation: Pathophysiological Mechanisms and Therapeutic Implications. Am J Med 2018;131:1408-17.

10. Imazio M, Lazaros G, Picardi E, et al. Incidence and prognostic significance of new onset atrial fibrillation/ flutter in acute pericarditis. Heart 2015;101:1463-7.

11. Kirchhof P, Benussi S, Kotecha D, et al. 2016 ESC Guidelines for the management of atrial fibrillation developed in collaboration with EACTS. Eur Heart J 2016;37:2893-962.

12. Lévy S, Breithardt G, Campbell RW, et al. Atrial fibrillation: current knowledge and recommendations for management. Working Group on Arrhythmias of the European Society of Cardiology. Eur Heart J 1998;19:1294-320.

13. Wardlaw JM, Smith EE, Biessels GJ, et al. Neuroimaging standards for research into small vessel disease and its contribution to ageing and neurodegeneration. Lancet Neurol 2013;12:822-38.

14. Calkins H, Hindricks G, Cappato R, et al. 2017 HRS/ EHRA/ECAS/APHRS/SOLAECE expert consensus statement on catheter and surgical ablation of atrial fibrillation. Europace 2018;20:e1-160.

15. Calkins H, Kuck KH, Cappato R, et al. 2012 HRS/EHRA/ ECAS expert consensus statement on catheter and surgical ablation of atrial fibrillation: recommendations for patient selection, procedural techniques, patient management and follow-up, definitions, endpoints, and research trial design.
J Interv Card Electrophysiol 2012;33:171-257.

16. Dong JZ, Sang CH, Yu RH, et al. Prospective randomized comparison between a fixed '2C3L' approach vs. stepwise approach for catheter ablation of persistent atrial fibrillation. Europace 2015;17:1798-806.

17. Miranda WR, Oh JK. Effusive-Constrictive Pericarditis. Cardiol Clin 2017;35:551-8.

18. Njoku A, Kannabhiran M, Arora R, et al. Left atrial volume predicts atrial fibrillation recurrence after radiofrequency ablation: a meta-analysis. Europace 2018;20:33-42.

19. Fu L, Rao F, Lian F, et al. Mechanism of electrical remodeling of atrial myocytes and its influence on susceptibility to atrial fibrillation in diabetic rats. Life Sci 2019;239:116903.

20. Kawakami H, Ramkumar S, Nolan M, et al. Left Atrial Mechanical Dispersion Assessed by Strain Echocardiography as an Independent Predictor of NewOnset Atrial Fibrillation: A Case-Control Study. J Am Soc Echocardiogr 2019;32:1268-1276.e3.

21. Nattel S, Harada M. Atrial remodeling and atrial fibrillation: recent advances and translational perspectives. J Am Coll Cardiol 2014;63:2335-45.

22. Amerena J, Ridley D. An Update on Anticoagulation in Atrial Fibrillation. Heart Lung Circ 2017;26:911-7.

23. Kalantarian S, Ay H, Gollub RL, et al. Association between atrial fibrillation and silent cerebral infarctions: a systematic review and meta-analysis. Ann Intern Med 2014;161:650-8.

24. Graff-Radford J, Gunter JL, Knopman DS, et al. Atrial fibrillation, cognitive impairment, and neuroimaging. Alzheimers Dement 2016;12:391-8.
Cite this article as: $\mathrm{Yu} \mathrm{R}, \mathrm{Xi} \mathrm{H}$, Wang $\mathrm{P}, \mathrm{Xu} \mathrm{D}, \mathrm{Lu} \mathrm{J}, \mathrm{Xu} \mathrm{F}$, An L, Zhao X, Bai R. Catheter ablation of atrial fibrillation after pericardiectomy: multi-center experience in China. Ann Transl Med 2020;8(9):580. doi: 10.21037/atm.2020.04.49 